\title{
Simultaneous dual-color fluorescence microscope: A characterization study
}

\author{
Zheng $\mathrm{Li}^{\mathrm{a}}$, Xiaodong Chen ${ }^{\mathrm{b}}$, Liqiang Ren ${ }^{\mathrm{a}}$, Jie Song ${ }^{\mathrm{a}, \mathrm{c}}$, Yuhua $\mathrm{Li}^{\mathrm{a}}$, Bin Zheng ${ }^{\mathrm{a}}$ and Hong $\mathrm{Liu}^{\mathrm{a}, *}$ \\ ${ }^{a}$ School of Electrical and Computer Engineering, University of Oklahoma, Norman, OK, USA \\ ${ }^{\mathrm{b}}$ College of Precision Instruments and Opto-electronics Engineering, Tianjin University, Tianjin, China \\ ${ }^{\mathrm{c}}$ Department of Biology, Mudanjiang Medical University, Mudanjiang City, China
}

Received: January 8, 2014

Accepted: May 11, 2014

\begin{abstract}
.
BACKGROUND: High spatial resolution and geometric accuracy is crucial for chromosomal analysis of clinical cytogenetic applications. High resolution and rapid simultaneous acquisition of multiple fluorescent wavelengths can be achieved by utilizing concurrent imaging with multiple detectors. However, such class of microscopic systems functions differently from traditional fluorescence microscopes.

OBJECTIVE: To develop a practical characterization framework to assess and optimize the performance of a high resolution and dual-color fluorescence microscope designed for clinical chromosomal analysis.

METHODS: A dual-band microscopic imaging system utilizes a dichroic mirror, two sets of specially selected optical filters, and two detectors to simultaneously acquire two fluorescent wavelengths. The system's geometric distortion, linearity, the modulation transfer function, and the dual detectors' alignment were characterized.

RESULTS: Experiment results show that the geometric distortion at lens periphery is less than $1 \%$. Both fluorescent channels show linear signal responses, but there exists discrepancy between the two due to the detectors' non-uniform response ratio to different wavelengths. In terms of the spatial resolution, the two contrast transfer function curves trend agreeably with the spatial frequency. The alignment measurement allows quantitatively assessing the cameras' alignment. A result image of adjusted alignment is demonstrated to show the reduced discrepancy by using the alignment measurement method.

CONCLUSIONS: In this paper, we present a system characterization study and its methods for a specially designed imaging system for clinical cytogenetic applications. The presented characterization methods are not only unique to this dual-color imaging system but also applicable to evaluation and optimization of other similar multi-color microscopic image systems for improving their clinical utilities for future cytogenetic applications.
\end{abstract}

Keywords: Fluorescence microscopy, medical imaging, imaging systems

\section{Introduction}

Thanks to the continuous research efforts in investigating the mechanism of cancer development in the last 40 years, the association between chromosome changes and transformation of normal human cells into cancer cells has been well discovered and better understood [1]. As a result, cytogenetic diagnostic methods of chromosome analysis are widely used in

*Corresponding author: Hong Liu, School of Electrical and Computer Engineering, University of Oklahoma, 110 West Boyd Street, Devon Energy Hall 150, Norman, OK 73019, USA. Tel.: +1 405325 4286; Fax: +1 405325 7066; E-mail: liu@ou.edu. biological researches and clinical practices. Among them, fluorescence in situ hybridization (FISH) has demonstrated its utility in localizing and quantifying DNAs in chromosomes for investigating association between chromosomal abnormalities and pathological developments as well as for confirming abnormalities found by other assays. A fluorescence microscope is an optically modified light microscope that enables detecting objects at molecular scale through amplified FISH signals [2]. Modern advances in fluorescence probe, optic filter, detector, and computation technology lead to significantly improved image quality and resolution of fluorescence microscopes and, therefore, 
overall accuracy for cytogenetic applications such as for cancer diagnosis and prognosis assessment.

Automated whole slide scanning to generate digital microscopic slides is an essential element of digital pathology [3]. Most commercial available wide-field FISH systems rely on switching motorized filter wheels for acquiring multiple FISH wavelengths during the slide digitization process. However, this practice is inefficient for generating images of multiple FISH channels due to the necessity of imaging every field of view (FoV) multiple times, especially when the depth of focus (DoF) is limited where acquisition of multiple image planes is required. In addition, temporal discrepancy will be introduced by consecutive image acquisitions in the situations of real-time imaging. To retrieve multi-spectral images, various imaging technologies were applied to microscopy such as liquid crystal tunable filter (LCTFs), acousto-optical tunable filter (AOTF) [4] and Fourier transform interferometry [5]. Confocal microscopes are specialized in imaging thick specimens [6] and capable of spectral imaging [7]. Outof-focus light is reduced by optical sectioning, which results in superior contrast and axial resolution compared to conventional epi-fluorescence microscopes [8]. As the effect of point spread functions is reduced, optical sectioning in theory improves lateral resolution as well [9]. However, galvanometer-based confocal microscopy sacrifices acquisition speed, and its image scanning is a rather slow process. As an improvement as well as a commercially available product, resonant scanning confocal microscopy [10] is able to achieve fast, video-rate scanning speed. Possibly due to manufacture costs of the high-precision optical and electronic components, a commercialized confocal microscope is much more expensive than a wide-field fluorescence microscope, and the former is therefore a less appealing solution for many laboratories. As a result, conventional wide-field fluorescence microscopes have been remaining as a popular and practical imaging tool for clinical FISH examination [11].

Various attempts that aim at simultaneously imaging multiple FISH wavelengths utilizing multiple detectors have been made, both academically and commercially. Using a dichroic mirror to split the emitted light and two detectors to capture image at video rate, Morris et al. demonstrated the feasibility of real-time multiwavelength fluorescence imaging [12]. Similar design was made with four fluorescent channels [13]. In recent years, a number of simultaneous multi-wavelength microscopic imaging systems have been developed by manufactures including Andor, Cairn Research, Hamamatsu, and Photometics. Dual detectors were also used in multifocal plane microscopy to track in vivo fluorescence signals spanning across multiple focal planes [14]. However, using multiple detectors can introduce a number of problems that not exist do for conventional microscopes. For example, separate light path can create various discrepancies among the images such as registration error and magnification difference. For applications where high spatial resolution and geometric accuracy are required, such kinds of problems may lead to bias or even erroneous results and may potentially compromise diagnoses and treatments. In order to validate a FISH imaging system that was specifically designed for clinical chromosomal analysis, we conducted this characterization study to systematically assess its performance. In addition, the presented characterization methods can also be applied on other systems that follow the similar principle.

\section{Materials and methods}

\subsection{System description}

A picture of the system, which is based on a Nikon Eclipse 50i wide-field microscope, is shown in Fig. 1b. Its optical principle is illustrated in Fig. 1a. The light source is a $200 \mathrm{~W}$ metal-halide lamb (Lumen 200, Prior Scientific, MA). Two fluorescence filter sets are installed. The first filter set inside the microscope's filter chamber consists of a multi-band excitation filter (XF1053 405-490-555650QBEX, Nikon) and a multi-band dichroic mirror (XF2046 400-485-558-640QBDR, Nikon). The original binocular was removed and replaced with a second filter set, which is for splitting the emission light to reach respective detectors. The second filter set is composed of a dichroic mirror and two emission filters for DAPI (QMAX EM 420-480, QuantaMAX) and spectrum orange (XF3022 580DF30, Nikon). Each emission filter allows only one band to pass onto a CCD detector to produce a monochromatic image. In the current setting, only the images of blue DAPI and spectrum orange are acquired. For this characterization study, three Nikon Plan Apo $\lambda$ series objective lenses $(10 \times / 0.45,60 \times / 0.95$, and $100 \times / 1.45)$ are utilized.

The two CCD detectors (CM-141MCL, JAI Inc., Japan) used in the microscope have a pixel size of 
(a)

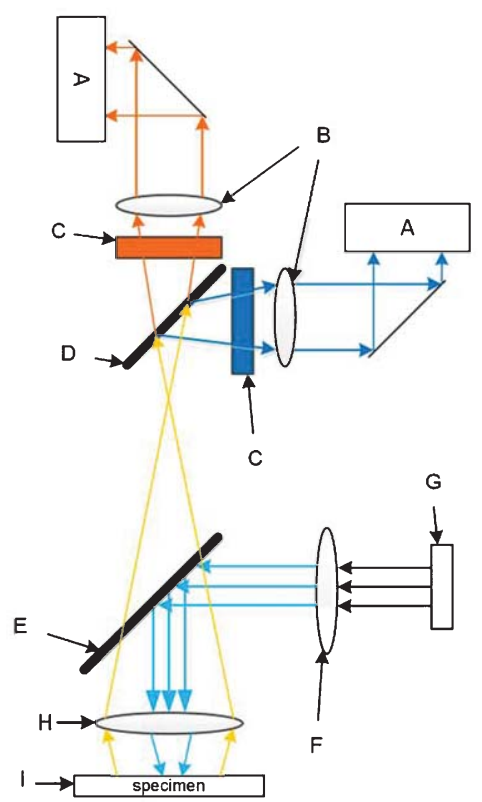

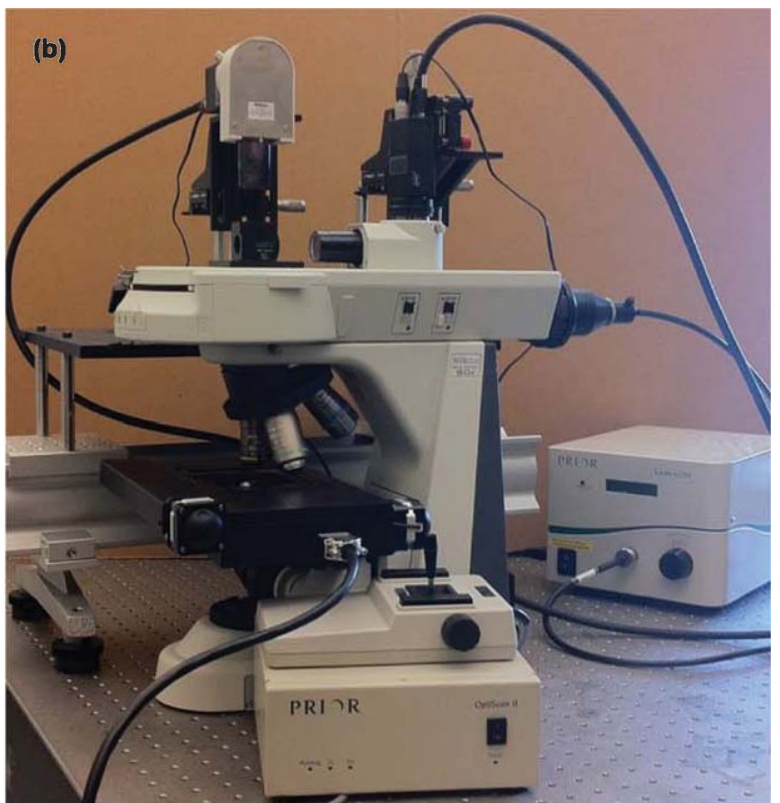

Fig. 1. (a) A schematic draw of the system's optics. The collimated beam from a 200W metal-halide source first passes through a multi-band excitation filter and reflected by a multi-band dichroic mirror toward the specimen. A second single-band dichroic mirror splits the transmitted emission light into two separate beams for simultaneous detection. (b) The picture of the microscope system.

$6.45 \mu \mathrm{m} \times 6.45 \mu \mathrm{m}$ and a pixel array of $1392 \times 1040$. Each CCD detector is coupled with a $0.7 \times$ relay lens (C-TEP, Nikon, Japan) and a PCI frame grabber (X64-CL iPro, DALSA, Canada) to compose an image acquisition unit. Each unit is joined with a set of adjustable optical stages for positioning and orienting the detector. An adjustable stage set includes a high-performance low-profile ball bearing linear stage (model 423, Newport, VA), 3 SM-13Vernier micrometers (Newport, VA) and a Techspec kinematic table platform (Edmund, NJ) as shown in Fig. 2. The reason for adjusting position and orientation of the detector will be discussed in later section. A high-precision programmable motorized stage (Model OptiScan II, PRIOR, UK) is mounted in the microscope. The motorized stage and the image acquisition components are connected to a host computer for integrated control for image acquisition and scan.

\subsection{System characterization}

Since the presented imaging system was specifically designed for chromosome analysis of clinicl cytogenetics, it has to meet a number of particular requirements. First of all, studying genomic loci and their transcriptional activities requires high spatial resolution, which is described by the system's contrast transfer function (CTF). Furthermore, high geometric accuracy is mandatory. In addition to geometric distortion, which is the primary cause of geometric inaccuracy in single detector microscopy, registration error and magnification difference induced by improperly aligned detectors can also compromise the validity of data in situations such as analyzing radial position of genomic loci. In order to validate the feasibility of the system, the following performances of the system: geometric distortion, photon signal linearity, CTF, and dual camera alignment were characterized.

\subsubsection{Geometric distortion}

Geometric distortions that exist in almost all optical systems distort the spatial relationship in the microscope images. Geometrically distorted images result in changed shape and size of objects. The distortion may be measured by observing the difference in pixel of a uniform distance between two objects at the center versus at the edge of the image.

If a perfect crossed scale without any mechanical error were imaged by a distortion-free imaging system, the marks on the scale would progress linearly from 


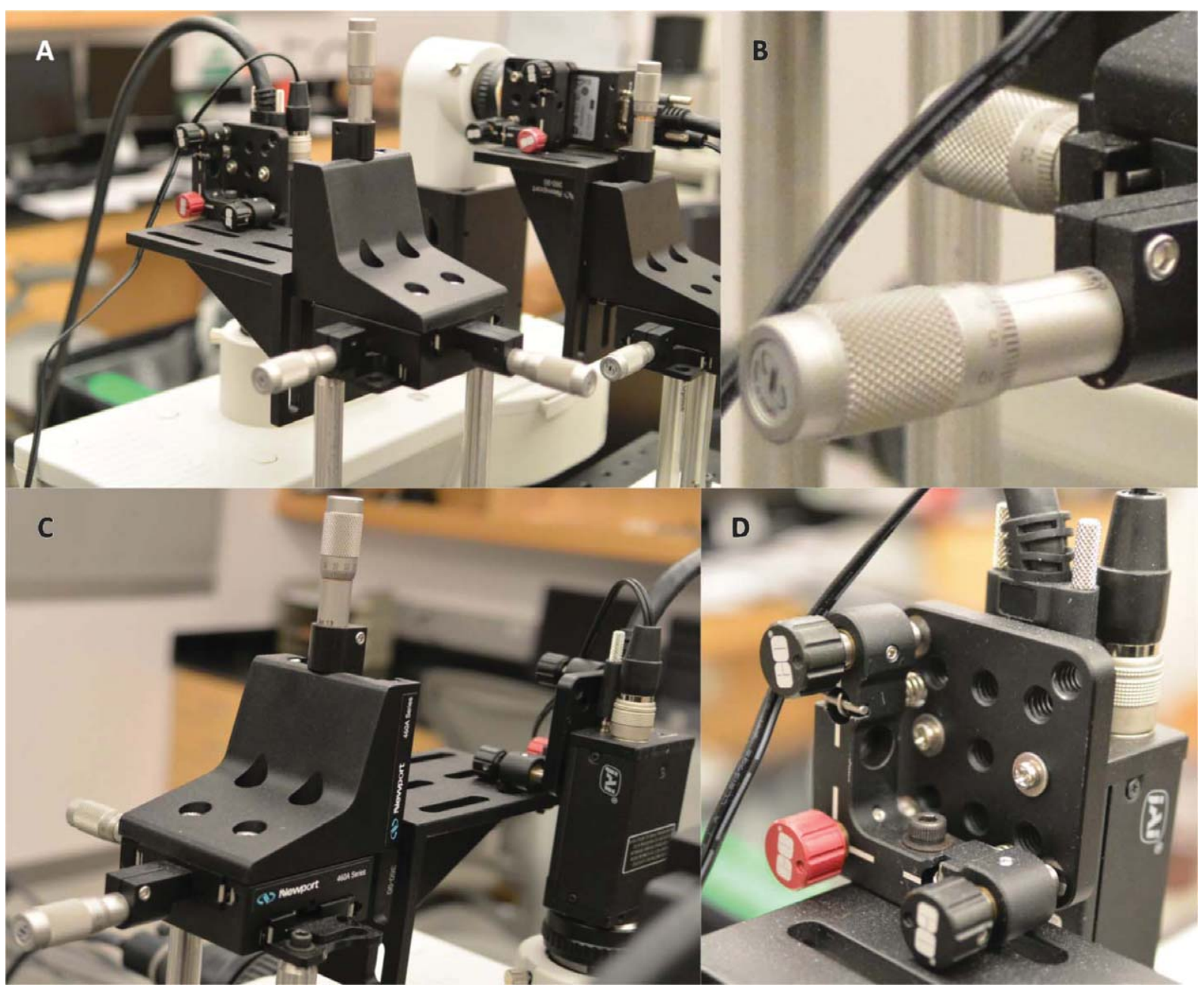

Fig. 2. Pictures of the alignment module. (A) Each camera/relay lens pair is coupled with a set of components for position adjustment. (B) SM13 Vernier micrometers. (C) a low-profile ball bearing linear stage with 3 micrometers. (D) A Techspec kinematic table platform for orientation adjustment.

the geometric center toward the peripheries. In reality, because of distortions, the marks progress nonlinearly instead. The distortion is measured by comparing the observed distance against a standard distance, which can be approximated by multiplying the unit distance at the lens center where the geometric distortion is minimal, using the following equations:

$$
\begin{aligned}
& D_{i}=\frac{r_{i}^{\prime}-r_{i}}{r_{i}} \times 100 \% \\
& r_{i}^{\prime}=\frac{1}{4} \sum_{j=1}^{4}\left\|p_{j i}-p_{0}\right\|
\end{aligned}
$$

$$
r_{i}=i \times r_{1}^{\prime}
$$

where $p_{j i}$ is an indexed mark with the direction index $j$ and the mark index $i$ on each of the directions, $p_{0}$ the center of the scale, $\left\|p_{j i}-p_{0}\right\|$ the distance from $p_{j i}$ to the center, $r_{i}^{\prime}$ the observed distances, $r_{1}^{\prime}$ the standard interval, $r_{i}$ the standard distance, and $D_{i}$ the distortion in percentage.

\subsubsection{Linearity}

Linear relationship between the input light photon and the output digital intensity is important for accurate acquisition of fluorescence signals. A detector's linearity profile can be measured by recording 

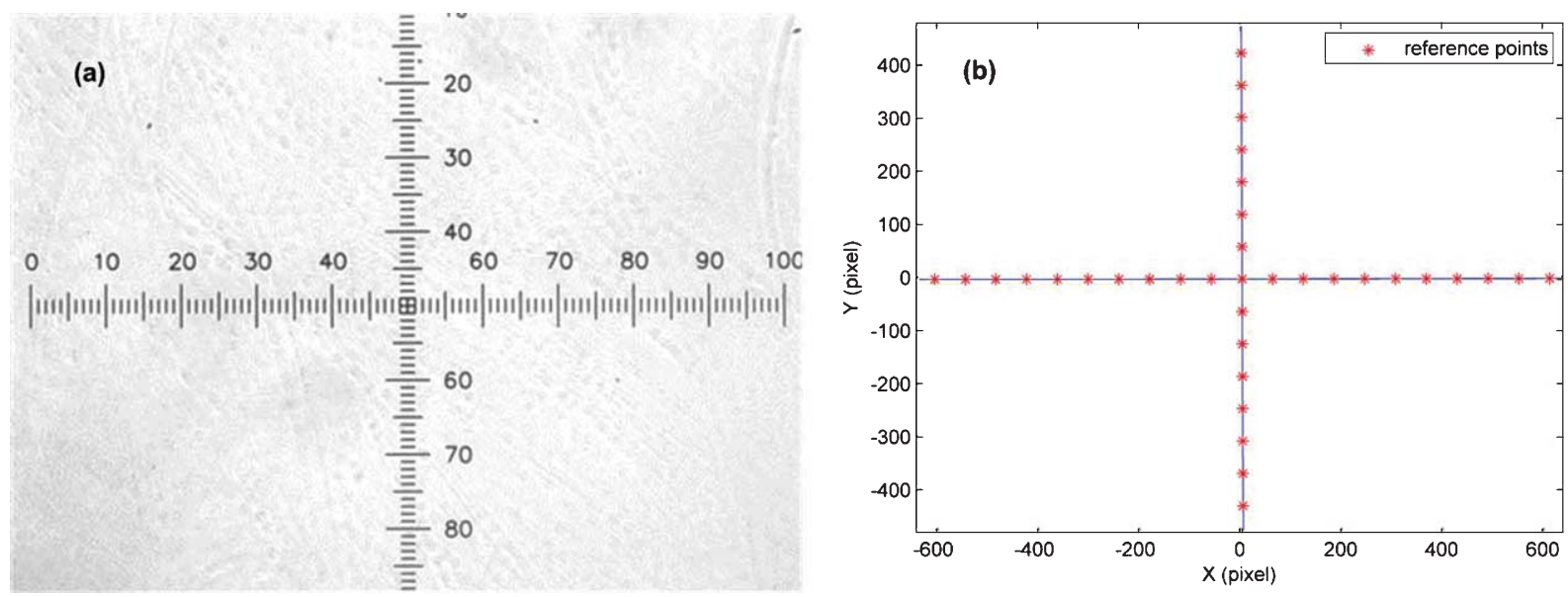

Fig. 3. Illustration for calculating the geometric distortion. (a) An example image of the $1 \mathrm{~mm}$ in $0.01 \mathrm{~mm}$ divisions crossed micrometer scale target, taken under the $10 \times$ objective lens. (b) The corresponding map of the marks, which are the geometric centers of the marks. The blue lines represent the distance from each mark to the scales' center.

(a)

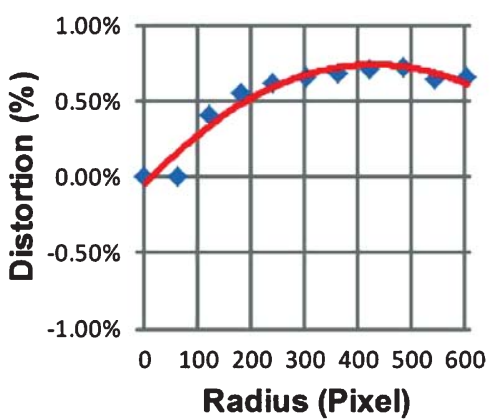

(b)

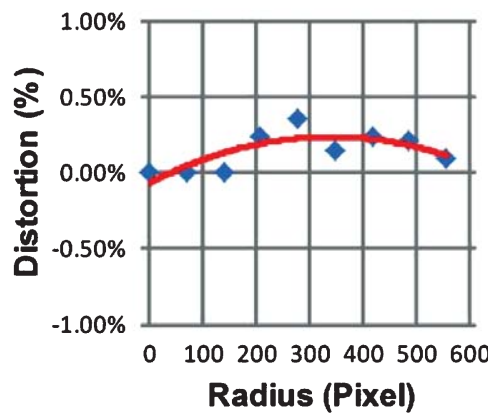

(c) $\times 100$

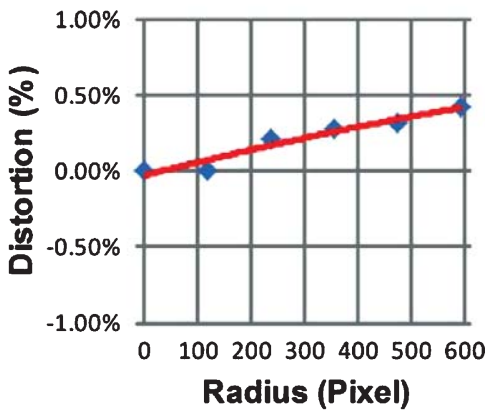

Fig. 4. Distortion profiles of the system under different objective lenses: (a) $10 \times / 0.45$ Nikon Plan Apo $\lambda$ objective lens. (b) $60 \times 60 \times / 0.95$ Nikon Plan Apo $\lambda$ objective lens. (c) $100 \times / 1.45$ Nikon Plan Apo $\lambda$ oil-immersion objective lens.

the change in output digital grayscale intensity versus the change in the input intensity or the integration time. The intensity may be controlled by applying a standardized microscopic slide of different transmission scales and differentiating the integration time. The overall transmitted intensity is governed by the following equations:

\section{Transmitted intensity}

$$
=\text { transmission } \times \frac{\text { controlled integration time }}{\text { maximum integration time }}
$$

Maximum integration time

$$
=\frac{1}{\text { frame rate }}-\text { frame transfer time }
$$

\subsubsection{Spatial resolution and contrast transfer function}

CTF is useful for evaluating properties of an optical system. Targets of periodic line grating at different spatial frequencies can be used to measure the CTF [15]. The CTF is calculated using the following equation:

$$
C T F=\frac{I_{\max }-I_{\min }}{I_{\max }+I_{\min }}
$$

where $I_{\max }$ and $I_{\min }$ are maximum and minimum pixel intensity of each line pair, respectively.

\subsubsection{Detector alignment}

When the two cameras are not aligned properly, there will be geometric discrepancy between the images from the two cameras. More importantly, the 
(a) Linearity of CCD1

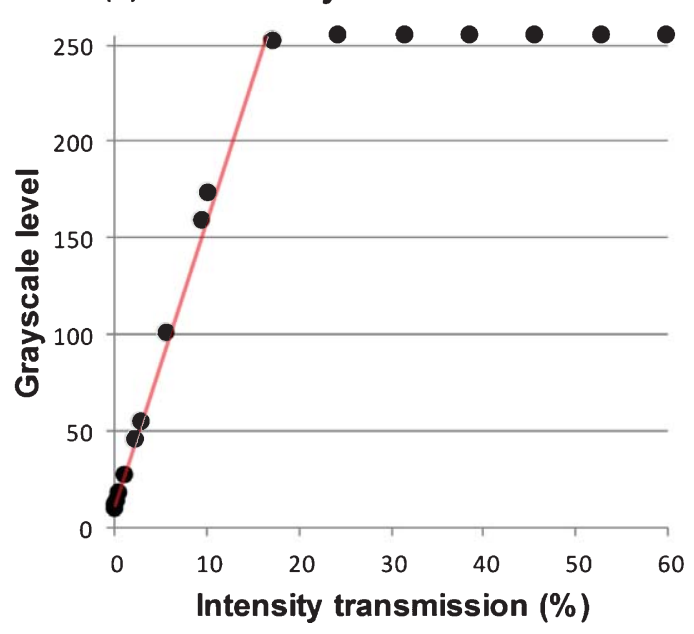

(b) Linearity of CCD2

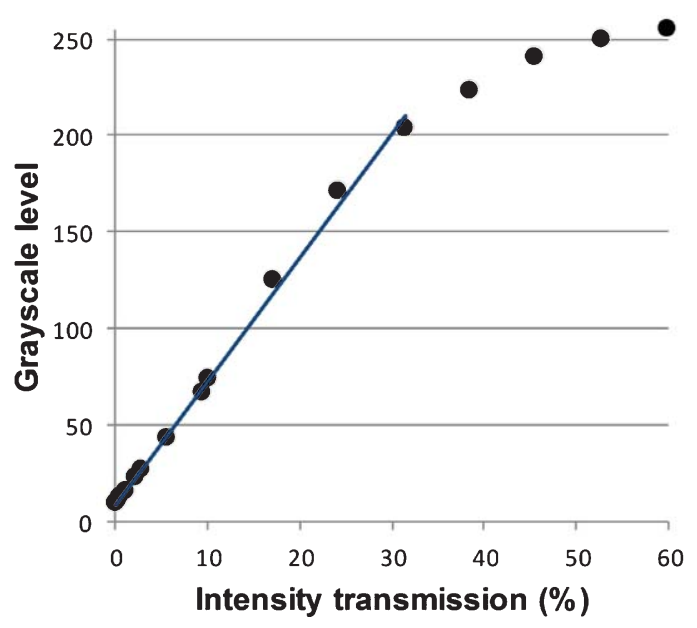

Fig. 5. The linearity profiles of the CCD detectors. The profiles are plotted as intensity transmission versus grayscale levels demonstrated linear but varied profiles before reaching the saturation point.

FISH signals may incorrectly show as outside the cell boundary, which would lead to erroneous and misleading data. This is because each detector has its own image plane, and the incident light beams strike on the two image planes differently, with different incident locations and angles. This section will discuss the method we used to measure the alignment of the two detectors so the difference, between both the incident locations and angles, can be minimized.

Suppose camera 1's image plane is plane xy, then the three perpendicular planes, $x y, y z$, and $x z$, are referred as the reference planes. The angle between the two cameras' image planes, which will be referred as the relative rotation, is approximated in terms of the angles in plane $\mathrm{xy}, \mathrm{yz}$, and $\mathrm{xz}$, denoted as $\psi, \alpha$, and $\beta$, respectively. A cross standard pattern is used to measure the relative rotation and the translocation. The cross standard pattern has a horizontal line and a vertical line, on which there are certain number of marks with an uniform distance between each two adjacent marks. The translocation of the cross center and the translocation of each mark's centroid pixel are both quantities that must be considered in order to minimize the overall translocation, which is expressed by the mean and the standard deviation of the centroids.

Suppose there are $n$ horizontal marks and $m$ vertical marks, and the centroids of the vertical marks fit into a line $p_{1}$ and the centroids of horizontal marks fit a line $p_{2}$. Using the prime symbol to distinguish camera 2 from camera $1, \psi$ is calculated as:

$$
\tan \psi=\frac{1}{2} \times\left(\frac{p_{1}-p_{1}^{\prime}}{1+p_{1} p_{1}^{\prime}}+\frac{p_{2}-p_{2}^{\prime}}{1+p_{2} p_{2}^{\prime}}\right)
$$

Suppose $\alpha$, and $\beta$ are very small, we can estimate them by:

$$
\begin{aligned}
\cos \alpha= & \frac{\min \left(\left|y_{b_{1}}-y_{b_{m}}\right|,\left|y_{b_{1}}^{\prime}-y_{b_{m}}^{\prime}\right|\right)}{\max \left(\left|y_{b_{1}}-y_{b_{m}}\right|,\left|y_{b_{1}}^{\prime}-y_{b_{m}}^{\prime}\right|\right)} \\
\cos \beta= & \frac{\min \left(\left|x_{a_{1}}-x_{a_{n}}\right|,\left|x_{a_{1}}^{\prime}-x_{a_{n}}^{\prime}\right|\right)}{\max \left(\left|x_{a_{1}}-x_{a_{n}}\right|,\left|x_{a_{1}}^{\prime}-x_{a_{n}}^{\prime}\right|\right)}
\end{aligned}
$$

where $a_{i}, i=1,2, \ldots n$, are horizontal centroids and $b_{j}, j=1,2, \ldots m$, are vertical centroids; $\mathrm{x}$ and $\mathrm{y}$ are the horizontal and vertical coordinates.

\section{Results}

\subsection{Geometric distortion}

A $1 \mathrm{~mm}$ in $0.01 \mathrm{~mm}$ divisions crossed micrometer scales (product No. 2280-16, Ted Pella, Inc., United States. Accuracy within $0.001 \mathrm{~mm}$ ) was imaged under the three objective lenses (Fig. 3). The scale was placed in a way such that its origin coincides with the optical center. Geometric centers of the scale marks were used as reference points. Figure 4 shows the distortion curves under the three objective lenses. Whereas 

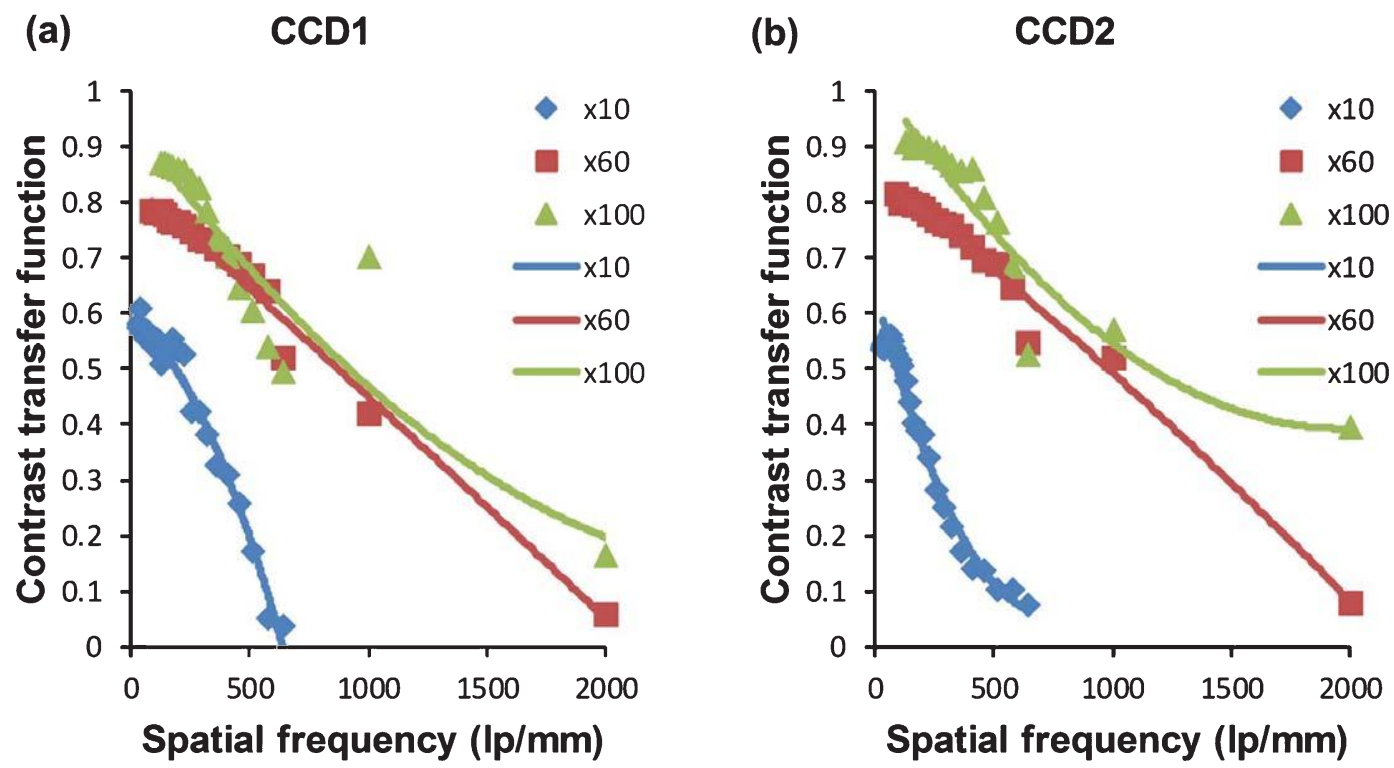

Fig. 6. Contrast transfer function (CTF) curves of the two CCD detectors. Two high resolution targets, USAF 2"X2" NEG (up to 6451p/mm, Edmund Industrial Optics, USA) and MRS-4 (up to 20001p/mm, Geller, USA), are imaged under the $10 \times$ and $60 \times$ objective lenses and the $100 \times$ oil objective lens, respectively. Each curve corresponds to an objective-CCD combination.

the horizontal axis represents marked distance to the image center, the vertical axis represents the corresponding distortion. The blue dots are locations where the measurement is made. Deviation from the $\mathrm{x}$-axis $(y=0)$ is the indication of geometric distortions. In spite of the non-monotonous increase from the center to the periphery, the system shows competent performance in terms of geometric distortion as the largest distortion values under the tested objectives lenses are below one percent, namely, $0.72 \%, 0.36 \%$ and $0.42 \%$, respectively.

\subsection{Linearity}

An EIA Grayscale Pattern slide (Edmund Optics, Barrington, $\mathrm{NJ}$ ) that has 9 equal step transmission rates $(3 \%, 10.125 \%, 17.250 \%, 24.375 \%, 31.500 \%$, $38.625 \%, 45.750 \%, 52.875 \%$ and $60 \%$ ) was used to profile the photon signal linearity property for both CCD detectors. The capture rate was set to 30 frames per second. A variation of integration time (1/60, $1 / 100,1 / 250,1 / 1000,1 / 1500,1 / 2000,1 / 4000)$ was combined with different transmission rates to control the transmitted photon intensity. The experiment was carried out in dark condition to avoid outside light. The profiles are plotted as functions of intensity transmission versus output grayscale levels in Fig. 5. Each detector shows good linearity characteristics, but their profiles differ in inclination and saturation due to variant attenuations of the filters and spectral response of the camera. Specifically, according to the camera's user's manual [16], the relative response ranges from $50 \%$ to $90 \%$ for wavelength between $420 \mathrm{~nm}$ and $480 \mathrm{~nm}$, compared to from $93 \%$ to $98.5 \%$ between $550 \mathrm{~nm}$ and $610 \mathrm{~nm}$. The disparity may be compensated by adjusting and matching the lookup tables.

\subsection{Spatial resolution and contrast transfer function}

Two high resolution periodic grating targets, USAF 2"X2" NEG (up to $645 \mathrm{lp} / \mathrm{mm}$, Edmund Industrial Optics, USA) and MRS-4 (up to 2000lp/mm, Geller, USA), were imaged to measure the CTF. The measurement was carried out for both CCD detectors under the three objective lenses, respectively. The normalized CTF curves are shown in Fig. 6.

\subsection{Detector alignment}

The microscopic target (Fig. 3a) used in distortion measurement was also utilized to measure the alignment. To demonstrate how the alignment was carried out using the proposed method, we use one regular 

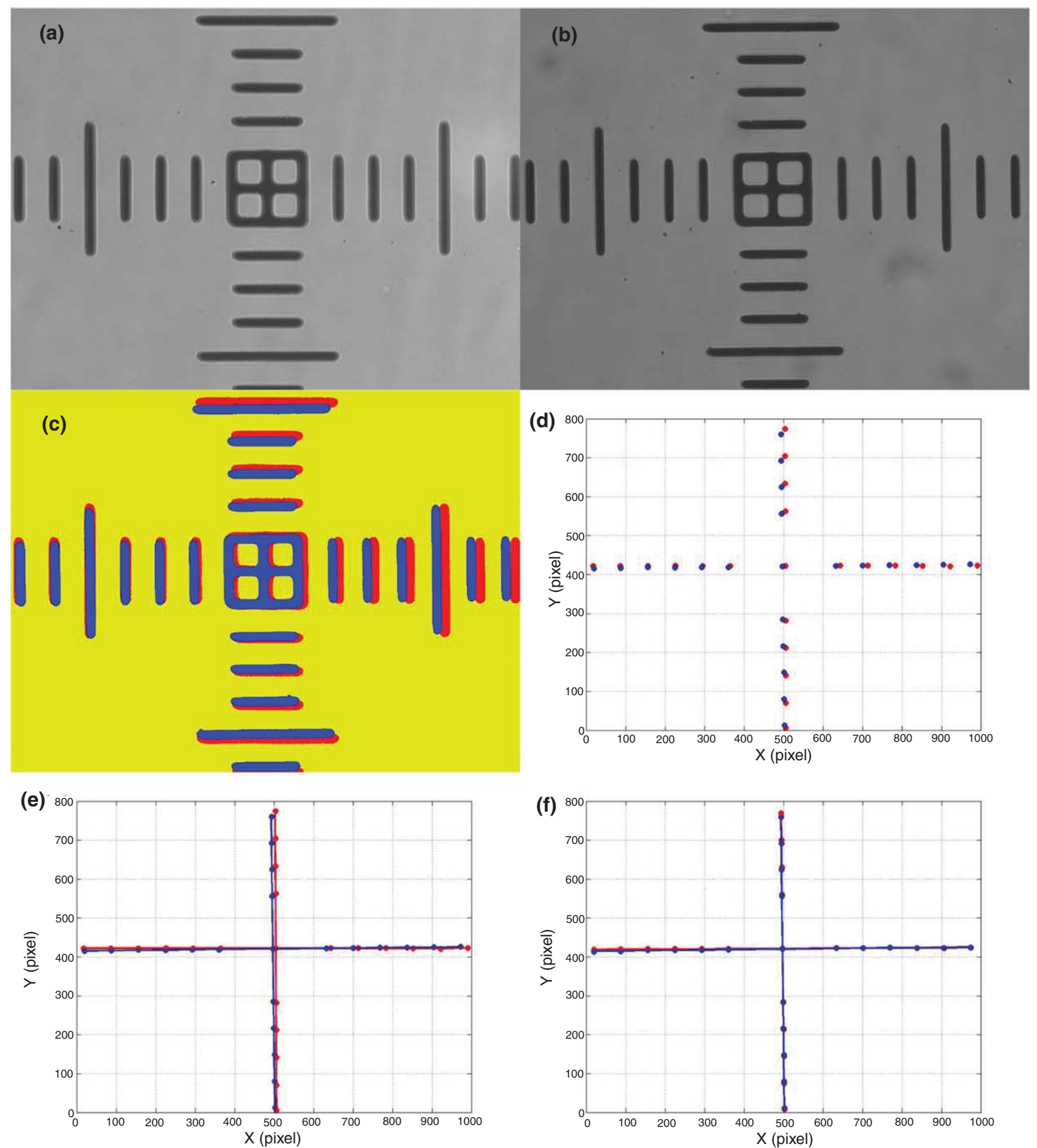

Fig. 7. Detector alignment measurement. The shown images were acquired under the $60 \times$ objective. (a) Image 1 taken by camera 1 . (b) Image 2 taken by camera 2. (c) Superimposed image 1 (red) and image 2 (blue). (d) The centroids of the marks. (e) The centroids with the perpendicular lines they fit into. (f) The centroids and the lines after the alignment by the proposed method.

calibration session as an example. The initial images are shown in Fig. 7(a) and (b), and their superimposed image is shown in Fig. 7(c). The centroids of the marks are plot in Fig. 7(d) and linearly fit into two cross lines as shown in Fig. 7(e), based on which the relative rotation $\psi, \alpha$, and $\beta$ and the translocations are 


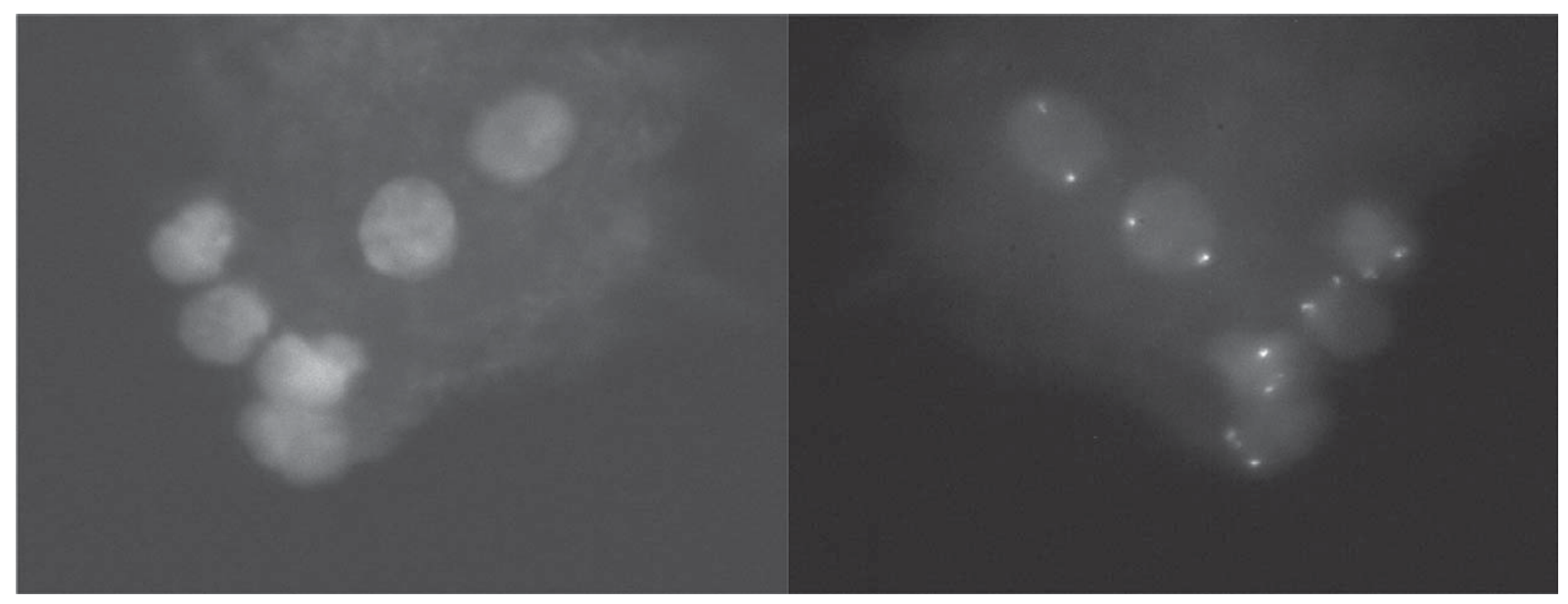

Fig. 8. FISH image taken by the presented system in real time (at 30 frame rate). On the left is the image of the DAPI (blue) and on the right is the image of the spectrum orange.

calculated. Adjusting the camera position and orientation based on the measurement result is a subjective and empirical procedure, which will be repeatedly applied with the measurement until the discrepancy is minimal. The result of the example session is shown in Fig. 7(f).

\section{Discussion}

A pair of FISH images acquired by the presented system is shown in Fig. 8. The presented characterization methods are important steps for applying the simultaneous dual-color microscopic imaging system in clinical chromosomal analysis in terms of achieving highest possible geometric accuracy at high resolution. Using the presented methods, the dual-color imaging system can be easily upgraded with faster and more sensitive detector to improve the performance including signal-to-noise ratio and acquisition efficiency. However, most chromosomal analyses for clinical purpose utilize at least two FISH probes, but the presented imaging system only has two color channels. Although it is possible to add more additional channels following the same principle, several considerations must be made. First, simultaneous excitation of multiple FISH probes may result in more spectrum overlapping. Color compensation [17] and profiling may be needed to mitigate the effect of signal crosstalk. Second, adding more dichromatic mirrors and emission filters may further reduce the number of photons per detector. As a result, faster and more sensitive detector may be needed to retain the current level of signal-to-noise ratio and/or frame rate.

\section{Acknowledgments}

This research is supported in part by National Institutes of Health (NIH) grants RO1 CA136700 and supported in part by a grant from the University of Oklahoma Charles and Peggy Stephenson Cancer Center funded by the Oklahoma Tobacco Settlement Endowment Trust. The authors would like to acknowledge the support of Charles and Jean Smith Chair endowment fund as well.

\section{References}

[1] L.J. Campbell, Evolution of Cytogenetic Methods in the Study of Cancer, in: Cancer Cytogenetics - Methods and Protocols, Humana Press, New York, NY, 2011, pp. 3-11.

[2] D.B. Murphy, Fluorescence Microscopy, in: Fundamentals of Light Microscopy and Electronic Imaging, Wiley-Liss, New York, NY, 2001, pp. 177-203.

[3] B. McCullough, Y. Xiaoyou, T. Monticello and M. Bonnefoi, Digital microscopy imaging and new approaches in toxicologic pathology, Toxicologic Pathology 32 (2004), 49-58.

[4] H.R. Morris, C.C. Hoyt and P.J. Treado, Imaging spectrometers for fluorescence and raman microscopy: Acousto-optic and liquid crystal tunable filters, Applied Spectroscopy 48(7) (1994), 857-866.

[5] Z. Malik, D. Cabib, R.A. Buckwald, A. Talmi, Y. Garini and S.G. Lipson, Fourier transform multipixel spectroscopy for quantitative cytology, Journal of Microscopy-Oxford $\mathbf{1 8 2}$ (1996), 133-140. 
[6] R.H. Webb, Theoretical basis of confocal microscopy, in: Methods in Enzymology, Confocal Microscopy, P.M. Conn, Ed., San Diego: Academic Press, 1999, pp. 3-20,

[7] T. Zimmermann, Spectral imaging and linear unmixing in light microscopy, in: Microscopy Techniques, J. Rietdorf, Ed. 2005, pp. $245-265$

[8] J.G. White, W.B. Amos and M. Fordham, An evaluation of confocal versus conventional imaging of biological structures by fluorescence light microscopy, J Cell Biol 105(1) (1987), $41-48$.

[9] C.J.R. Sheppard and T. Wilson, The image of a single point in microscopes of large numerical aperture. Proceedings of the Royal Society of London Series A, Mathematical and Physical Sciences 379(1776) (1982), 145-158.

[10] N. Callamaras and I. Parker, Construction of a confocal microscope for real-time $\mathrm{x}-\mathrm{y}$ and $\mathrm{x}-\mathrm{z}$ imaging, Cell Calcium 26(6) (1999), 271-279.

[11] G. Pajor, B. Kajtar, L. Pajor and D. Alpar, State-of-the-art FISHing: Automated analysis of cytogenetic aberrations in interphase nuclei, Cytometry Part A 81A(8) (2012), 649-663.

[12] S.J. Morris, Real time multi-wavelength fluorescence imaging of living cells, Society for Neuroscience Abstracts 16(1) (1990), 54-54.
[13] S.J. Morris, T.B. Wiegmann, L.W. Welling and B.M. Chronwall, Rapid simultaneous estimation of intracellular calcium and PH, Methods in Cell Biology 40 (1994), 183-220.

[14] S. Ram, P. Prabhat, J. Chao, E.S. Ward and R.J. Ober, High accuracy 3D quantum dot tracking with multifocal plane microscopy for the study of fast intracellular dynamics in live cells, Biophysical Journal 95(12) (2008), 6025-6043.

[15] J.M. de Bakker, F.J. van Capelle, M.J. Janse, A.A. Wilde, R. Coronel, A.E. Becker, K.P. Dingemans, N.M. van Hemel and R.N. Hauer, Reentry as a cause of ventricular tachycardia in patients with chronic ischemic heart disease: Electrophysiologic and anatomic correlation, Circulation 77(3) (1988), 589-606.

[16] Spectral Response, in User's Manual for CM-141MCL/ 141MCL-RA Digital Monochrome/Color Progressive Scan Camera, 2010, p. 42.

[17] C. Hyohoon, K.R. Castleman and A.C. Bovik, Color compensation of multicolor FISH images, Medical Imaging, IEEE Transactions on 28(1) (2009), 129-136. 


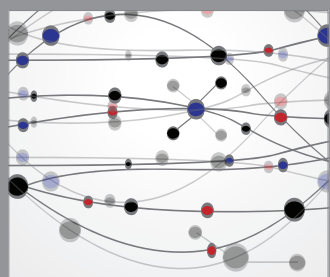

The Scientific World Journal
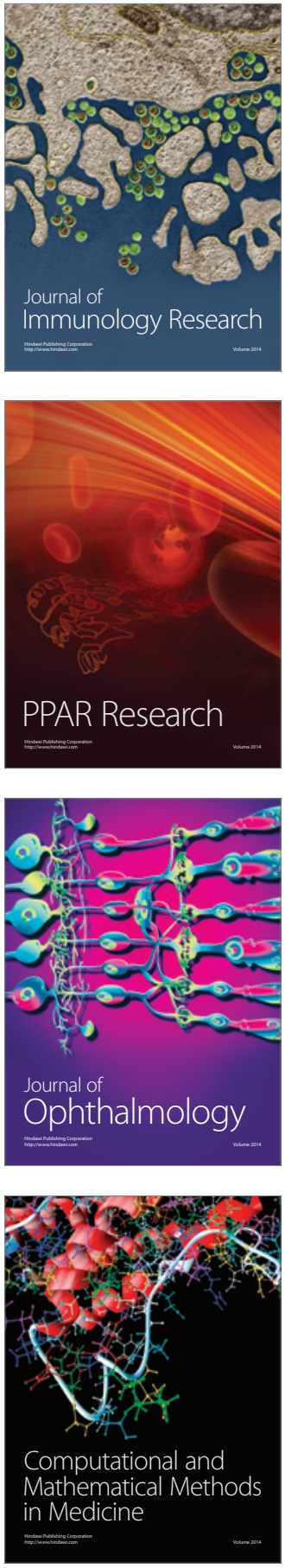

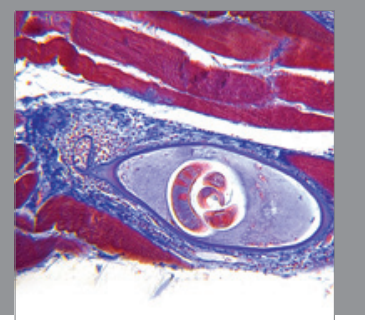

Gastroenterology

Research and Practice
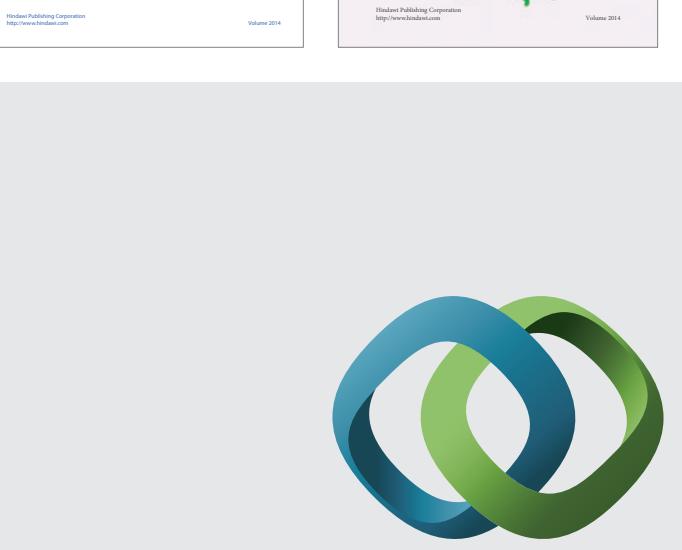

\section{Hindawi}

Submit your manuscripts at

http://www.hindawi.com
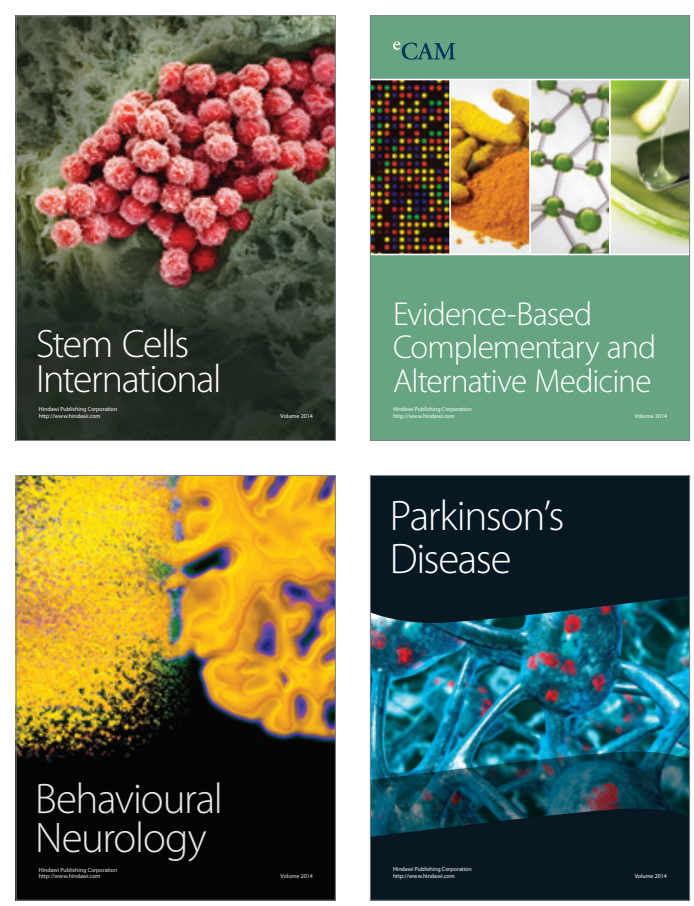

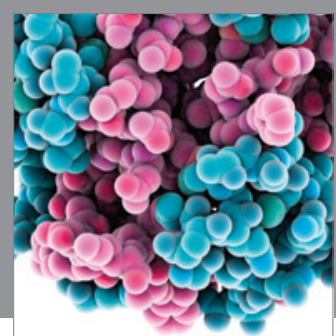

Journal of
Diabetes Research

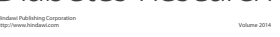

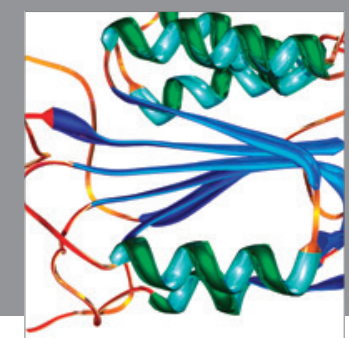

Disease Markers
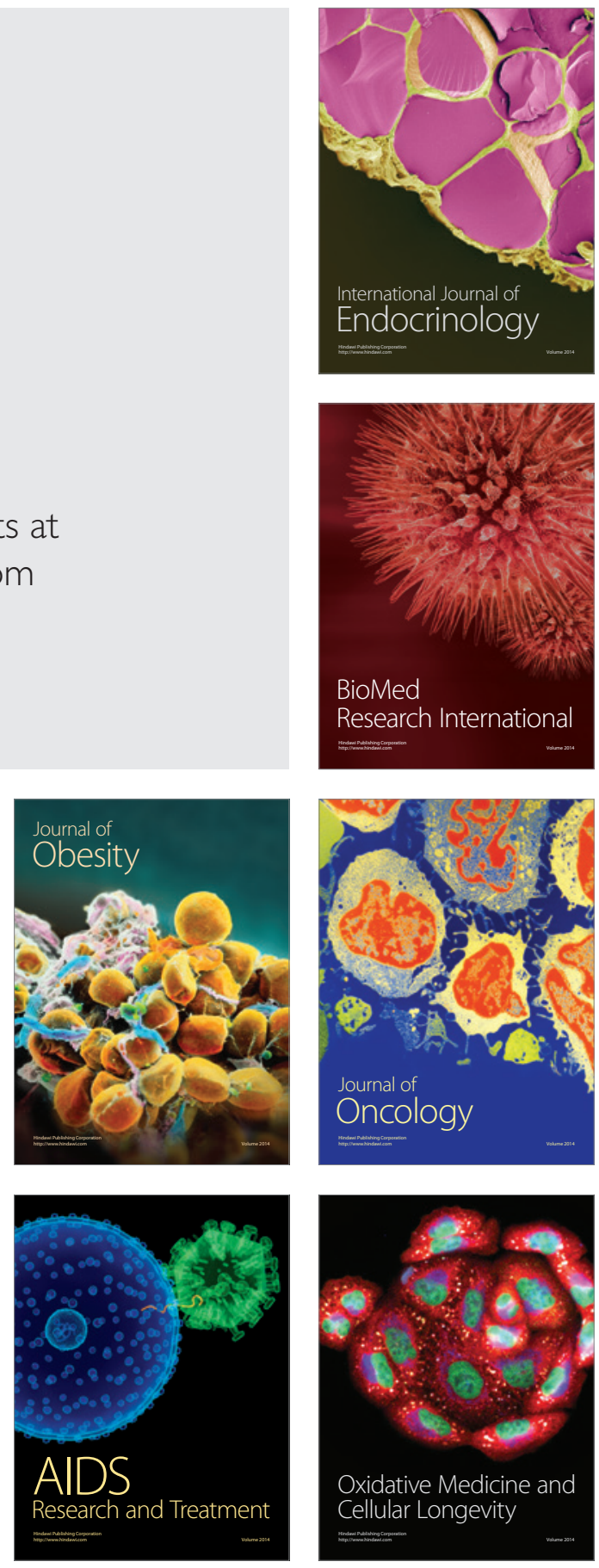